\title{
ASSESSMENT OF CARDIAC AUTONOMIC DYSFUNCTION AFTER PULMONARY VEIN ISOLATION AND ITS IMPACT ON SUCCESS OF PAROXYSMAL ATRIAL FIBRILLATION ABLATION.
}

\author{
Wagdy A. Galal, Mohamed Amin, Lamyaa Allam, Hassan Shehata,and \\ Mohamed A. Mousa
}

Department of cardiology,

Faculty of medicine - Ain shams

University

.Corresponding :

Mohamed Ahmed Ali Kotb Mobile: 01114228258

E mail:

dr.mohammed.mousa@gmail.com

Received: $13 / 6 / 2019$

Accepted: 16/7/2019

\begin{abstract}
Background: Atrial-fibrillation is the most prevalent cardiac arrhythmia and a significant public health issue. Recently, radiofrequency current ablation and cryoballoon ablation are frequently used for rhythm control in symptomatic paroxysmal AF patients who are resistant to anti-arrhythmic drugs or even as a firstline rhythm control strategy. Several studies have demonstrated an increase in heart rate and a decrease in heart rate variability after radiofrequency catheter ablation of atrial fibrillation due to vagal denervation.
\end{abstract}

Aim of the work: to Assess the incidence and the value of post AF ablation cardiac autonomic dysfunction after Pulmonary vein isolation using radiofrequency and cryoballoon ablation techniques.

Patients and Methods: This study enrolled 40 patients who underwent paroxysmal AF ablation at Ain Shams University hospitals. Patients were randomized into 2 groups, Radiofrequency ablation guided by $3 D$ mapping (RF group; $n=20$ ) and Cryoballoon ablation using $2^{\text {nd }}$ generation balloons (CB group; $n=20$ ). Valid 24-hour Holter for heart rate variability analysis was done for each patient pre ablation, after 1 month, after 3 months and after 12 months. Statistical comparison between both groups regarding HRV changes and clinical outcome was done.

Results: The efficacy data in the form of freedom from AF at one year using radiofrequency technique was slightly higher than cryoballoon technique (75\% Vs 65\% respectively). However, this difference wasn't statistically significant (P-value 0.490). During the 3-point follow up of HRV parameters (month-1, month-3 and month12), changes in time domain and frequency domain parameters persisted for 12 months after both techniques, being more pronounced in RF group than CB group. The change in HRV parameters was in favour of vagal withdrawal and sympathetic dominance (there was a Significant decrease in SDNN, rMSSD and triangular index, on the other hand there was a significant increase in LF/HF).

Conclusion: Cardiac autonomic dysfunction_assessed by Changes in time domain and frequency domain heart rate variability parameters_persisted for 12 months after both techniques, being more pronounced in radiofrequency group than cryoballoon group.

Keywords: *ablation *arrhythmia *atrium *fibrillation *cardiac autonomic dysfunction *heart rate variability * ${ }^{*}$ cryoablation.

\section{INTRODUCTION:}

Pulmonary vein (PV) ablation is an effective preventive strategy for treating paroxysmal atrial fibrillation (AF), but its antiarrhythmic mechanisms remain unclear. ${ }^{(1)}$ Increased vagal tone is frequently involved in the onset of AF in patients with 
structurally normal hearts. ${ }^{(2)}$ Vagal denervation in a canine model prevents inducible $\mathrm{AF}, \underline{(3)}$ and long-term vagal denervation of the atria renders AF less easily inducible. ${ }^{(4)} \mathrm{HRV}$ contains a lot of information about the cardiovascular regulation, thus subtracting and analyzing of the information of HRV can assess the cardiac autonomic function in humans quantitatively. Therefore, HRV is a noninvasive method in detecting the autonomic function of the hearts. ${ }^{(5)}$ Several studies have demonstrated a decrease in HR variability and an increase in heart rate after radiofrequency catheter ablation of supraventricular tachycardia or atrial fibrillateion. ${ }^{(6,7)}$ Vagal attenuation was assumed to be an additional mechanism of the antiarrhythmic action of circumferential Pulmonary vein isolation. ${ }^{(1)}$ Most of studies demonstrated that focal ablation of PVs may result in a transient increase in HR and a transient decrease of time-domain and frequency-domain $\mathrm{HRV}$, which indicates autonomic dysfunction (enhanced sympathetic activity or parasympathetic nervous withdrawal). ${ }^{(1,7,8)}$ Although autonomic dysfunction may play an important role in changes in HR and HRV after ablation of slow pathway, postero-septal space, and PVs, the precise mechanism is not clear. An increase of sympathetic tone, a decrease of parasympathetic tone, or a combination of both factors is the possible explanations. $\left.{ }^{(7,}, 9\right)$ Therefore, in this study patients undergoing radiofrequency $\mathrm{PV}$ ablation (RF) or cryoballoon ablation (CB) for paroxysmal $\mathrm{AF}$, were evaluated for the effects of RF and $\mathrm{CB}$ ablation on $\mathrm{PV}$ innervation, heart rate variability (HRV), and recurrence of AF.

\section{AIM OF THE WORK:}

1- Assess the incidence and the value of post AF ablation cardiac autonomic dysfunction and its impact on recurrence after Pulmonary vein isolation while comparing between radiofrequency and cryoballoon ablation techniques.

2- Assess basic sinus rate and heart rate variability changes post AF radiofrequency ablation in comparison to AF cryoballoon ablation.

\section{PATIENTS AND METHODS:}

This study is a Single Centre, randomized clinical study, recruiting 40 consecutive patients from Ain Shams University hospitals, Cairo, Egypt with symptomatic drug refractory paroxysmal AF who underwent AF ablation. Patients were randomized into 2 groups; 20 patients underwent radiofrequency $\mathrm{AF}$ ablation guided by $3 \mathrm{D}$ mapping and another matched cohort of 20 patients who were ablated using the second-generation Cryo balloon from March 2017 till July 2018 and were followed up for 1 year after the PVI procedure. Among the RF group, the standard CARTO RF ablation catheter was used in 11 procedures while 9 patients were operated by the newer generation, contactforce-sensing catheters (smart touch technology).

\section{Inclusion and exclusion criteria:}

Patients with symptomatic paroxysmal $\mathrm{AF}$ who failed to respond to at least one of class I or III antiarrhythmic drugs (AADs) were included. AF was documented by 12 lead ECG and/or Holter monitoring. Patients were excluded from the study when left atrial or intracardiac thrombi were discovered by transoesophageal echocardiography (TOE), patients with diagnosis of persistent or permanent $\mathrm{AF}$, or previous pulmonary vein isolation (PVI). Other exclusion conditions include consent refusal, Manifest accessory pathway detected by standard surface ECG, history of prior cardiac surgery, Structural heart disease other than LV hypertrophy, such as significant valvular heart disease $\geq$ grade 2 , mitral valve prosthesis, aortic valve 
prosthesis, hypertrophic, ischemic, dilated cardiomyopathies and congenital heart diseases.

\section{Methods:}

After giving informed consent and approval of the ethical committee the selected patients were subjected to the following:

Thorough history taking with particular stress on: Nature, duration of symptoms, EHRA class, Age, gender, predisposing factors for AF, Frequency of symptoms and its effect on patient's quality of life, prior history of DC cardioversion, anti-arrhythmic drug history (types of medications used, duration, response to drugs whether by success or failure), underlying heart disease (e.g.: coronary heart disease, rheumatic heart disease, dilated cardiomyopathy, congenital heart disease, hypertensive heart disease), previous complication related to atrial fibrillation (e.g.: thrombo-embolic events, hospitalization due to rapid uncontrolled rate).

Complete physical examination: (general and local) with special emphasis on orthostatic hypotension (Orthostatic hypotension $(\mathrm{OH})$ has been formally defined by expert consensus as a fall in systolic blood pressure (SBP) of at least $20 \mathrm{~mm} \mathrm{Hg}$ and/or diastolic blood pressure (DBP) of at least $10 \mathrm{~mm} \mathrm{Hg}$ within $3 \mathrm{~min}$ of standing). (10)

Baseline 12-lead electrogram: (done before \& after the procedure) to assess rate, rhythm, baseline intervals, any conduction disturbances.

24-hour validated Holter recording for assessment of time domain and frequency domain HRV parameters was done and analyzed for each candidate after stopping anti arrhythmic medications for 5 half-lives at least.

Echocardiogram: for baseline measurements of LA, LV dimensions, systolic \& diastolic LV functions, valvular functions and Transesophageal Echocardiography: to exclude left atrial and left atrial appendage thrombi

Anticoagulation was either interrupted or uninterrupted. In the former case, the medication was discontinued 3 days before the procedure with shifting to subcutaneous enoxaparin $1 \mathrm{mg} / \mathrm{kg}$ every 12 hours to be withhold on the night of the procedure.

\section{Cryoballoon Ablation:}

Most procedures were carried out under conscious sedation. Sedation was achieved by IV bolus doses of fentanyl, midazolam and IV morphia for pain relief. Bilateral femoral vein access was obtained without ultrasound guidance. A decapolar linear catheter was positioned in the coronary sinus (CS) to document the atrial and ventricular activations, and to be used, later during the procedure, to pace the phrenic nerve. Surface ECG and bipolar intracardiac electrograms were filtered from 30 to 300 $\mathrm{Hz}$ and were stored continuously for further analysis. Trans-septal puncture (TSP) was performed guided by fluoroscopy, TOE and pressure monitoring. IV Heparin, 7000-9000 $\mathrm{U}$, was given and monitored by repeated sampling every 30 minutes to keep activated clotting time (ACT) above 300.

A 15 French deflectable sheath (Flexcath Advance, Medtronic, MN, USA) was introduced, over a long stiff wire positioned in the left superior pulmonary vein (LSPV), into the left atrium and kept facing the LSPV ostium. After establishment of the gas and electrical connections to the cryoconsole, insertion of the circular octapolar catheter (Achieve, Medtronic) into the inner lumen of the balloon via a threeway connection, the second-generation cryoballoon catheter (Arctic Front Advance, Medtronic) was advanced through the sheath. The Achieve mapping catheter was placed in the lumen of LSPV and the balloon followed to stay at the antrum of the LSPV 
where the sheath is slightly pulled back and the balloon inflated. The balloon was pushed gently to seal the PV ostium and pulmonary venogram confirmed the occlusion of the vein before freezing was started. The duration of the freeze was variable according to the operator and ranged from 180 to 240 seconds according to the rate of temperature drop, minimum temperature reached, degree of occlusion of the vein, and pulmonary vein potential (PVP) dissociation.

A minimum temperature of $-40^{\circ} \mathrm{C}$ to $50^{\circ} \mathrm{C}$ was considered adequate, with additional application delivered if temperature failed to decline to this level. In some cases, PVP was observed in real-time during freezing with delay and subsequent dissociation documented. After isolation, pacing from Achieve was done to test confirm exit block, and CS catheter, for entrance block. These steps were repeated for all four PVs. Before isolation of the right PVs, phrenic nerve function was documented by catheter stimulation at the right subclavian vein site and was monitored by palpating the strength of diaphragmatic excursion during freezing. At the end of the procedure, haemostasis at the groin was achieved by a purse-string suture followed by manual compression. Procedural and fluoroscopy times were recorded. Figures 1,2 and 3.

Figure 1): The cryoballoon in action. The first image from the left represent a pulmonary venogram in RAO view in LSPV during occlusion by the inflated balloon to look for any leaks. If no leak is observed, freezing is initiated, the second image showing the cryobaloon inflated at the ostium of LSPV in LAO view, the 3rd image represent the cryoballoon inflated at the ostium of LIPV. LSPV; left superior pulmonary vein, LIPV; left inferior pulmonary vein, CS; coronary sinus; $\mathrm{PV}$, pulmonary vein; $\mathrm{SCV}$, subclavian vein.
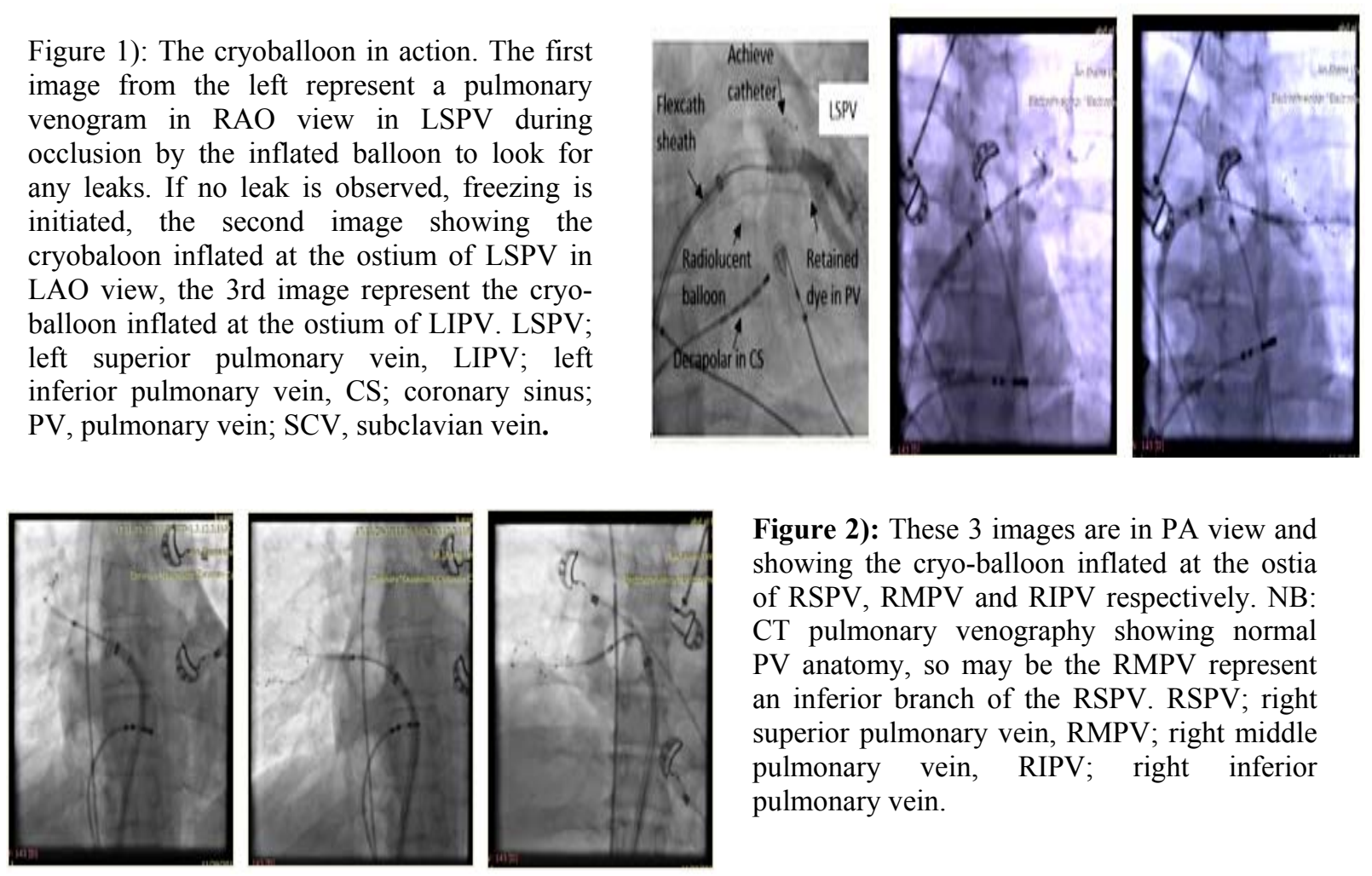

Figure 2): These 3 images are in PA view and showing the cryo-balloon inflated at the ostia of RSPV, RMPV and RIPV respectively. NB: CT pulmonary venography showing normal PV anatomy, so may be the RMPV represent an inferior branch of the RSPV. RSPV; right superior pulmonary vein, RMPV; right middle pulmonary vein, RIPV; right inferior pulmonary vein.

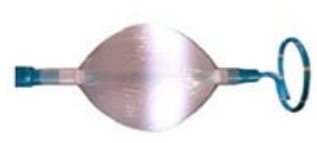

First-generation

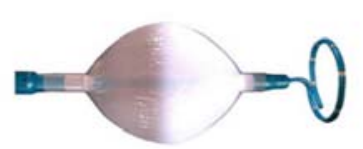

second-generation

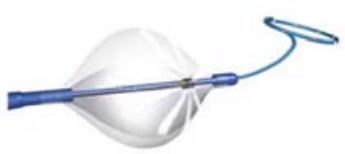

third-generation

Figure 3): Evolution of the cryoballoon. The second-generation balloon is the most widely used (courtesy: Medtronic) $(\underline{11})$ 


\section{Radiofrequency Ablation:}

All procedures were carried out under GA to prevent patient discomfort and avoid dislocation of the acquired electro anatomical maps of the CARTO 3 (Biosense Webster, CA, USA). Two transseptal punctures were performed to pass two long sheaths into the left atrium, one for the circular mapping catheter (Lasso, Biosense Webster), and the other for the $3.5 \mathrm{~mm}$ saline-irrigated ablation catheter (Thermocool, Biosense Webster). ACT was maintained above 300. Anatomical construction of the LA chamber was performed followed by circumferential PV antrum point-by-point ablation to encircle the ipsilateral left and right PVs. During ablation, the temperature limit was $40^{\circ} \mathrm{C}$, with RF energy of $25-30 \mathrm{~W}$, and infusion rate of $13-20 \mathrm{~mL} / \mathrm{min}$ with movement of the catheter tip along the line every 20 seconds. The power was reduced to $20-25 \mathrm{~W}$ at the LA posterior wall to avoid thermal injury of the oesophagus. Complete PV isolation was defined as the elimination of conduction of PV musculature in all pulmonary veins, as judged by either disappearance or dissociation of PVP. Confirmation of PVI was achieved by demonstration of either entrance block, defined as absence of PVP on the mapping catheter during pacing from a coronary sinus or a LA catheter, and exit block where pacing from the catheter in the PV doesn't cause atrial activity. Procedural and fluoroscopy times were recorded.

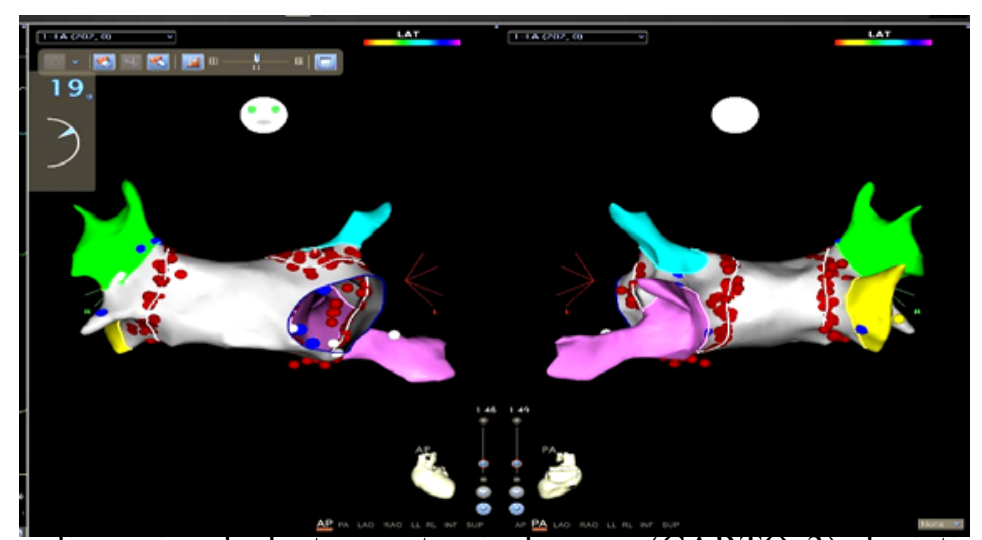

Figure 4): Three-dimensional electro-anatomical maps (CARTO 3) depicting the left atrium in an anteroposterior view (AP, left) and posteroanterior view (PA, right). The pulmonary veins are colored. The ablation lesions (red dots) are deployed in a circumferential manner around ipsilateral pulmonary veins (WACA, wide area of circumferential ablation).

\section{Post-ablation management and follow-up:}

Patient follow up was done at 1, 3 and 12 months after the procedure to detect AF recurrence and parameters for any cardiac autonomic dysfunction. The following data was collected: History taking as regards recurrence of the symptoms, complete physical examination as done before ablation procedure with special emphasis on orthostatic hypotension, surface ECG was taken 1,3 and 12 months after the procedure, holter monitoring in all patients 1,3 and 12 months after the procedure was done to evaluate basic sinus rate and HRV data and compare it with Holter data obtained before the procedure.

Cases were always asked to record their ECG when symptomatic. Any episode of symptomatic or asymptomatic atrial tachyarrhythmia that lasted more than 30 seconds documented with ECG or Holter monitoring was considered as a clinical recurrence after the first 3 months post ablation. 


\section{Holter data analysis:}

Heart rate variability parameters were analyzed by $24 \mathrm{~h}$ Holter monitoring device (NORAV medical ${ }^{\circledR}, \quad G m b h, \quad N H-301$, Germany). Recordings were taken at preand post-ablation 1, 3- and 12-months periods for each patient. Only high-quality recordings were considered for analysis. All recordings were reviewed by an experienced operator. Premature ventricular beats, premature atrial beats, and electrical artifacts were excluded from analysis. Heart rate variability parameters were used as indicators of autonomic activity according to previously published guidelines. ${ }^{(12)}$ the following parameters were documented for each recording: (Average HR in bpm, mean $\mathrm{RR}$ interval (mean NN interval) milliseconds, Standard deviation of $\mathrm{NN}$ interval (SDNN) in milliseconds, root mean square of differences between successive $\mathrm{NN}$ intervals (rMSSD) in milliseconds, Low frequency/high frequency ratio (LF/HF), triangular index. Post AF ablation high sinus rate (PA-HSR) was defined as an increase in resting sinus rate $\geq 20 \mathrm{bpm}$ or a resting sinus rate $\geq 92 \mathrm{bpm}$ without physiological or hemodynamic causes as defined in a cohort study made by Yu HT, et al. ${ }^{(13)}$ Comparison between mean sinus rate pre and post AF ablation was done. Heart rate variability changes (as an indicator of autonomic changes post AF ablation) were obtained and compared between radiofrequency and cryoballoon groups.

\section{Statistical analysis}

Analysis of data was done using SPSS software version 25 (IBM, Chicago, IL, USA). Quantitative variables were described as mean \pm standard deviation (SD), median and interquartile range. Qualitative variables were described as number and percentage. Independent t-test was used to compare quantitative variables in parametric data while Mann-Whitney test was performed for nonparametric variables. Comparison between groups as regards qualitative variables was done by using chi-square test. Fisher exact test was used instead of chisquare when one expected cell is less than 5 . The confidence interval was set at $95 \%$ and the accepted error margin was set at $5 \%$. The p-value was therefore regarded to be important as follows: $\mathrm{P}>0.05$ was nonsignificant, $\mathrm{P}<0.05$ was significant, and $\mathrm{P}<$ 0.001 was very significant.

\section{RESULTS}

A total of 40 patients were included in the analysis. Cryoballoon (CB) group consisted of 20 patients and the radiofrequency (RF) group comprised a sum of 20. Both groups were followed up at 1, 3and 12-months post AF ablation.

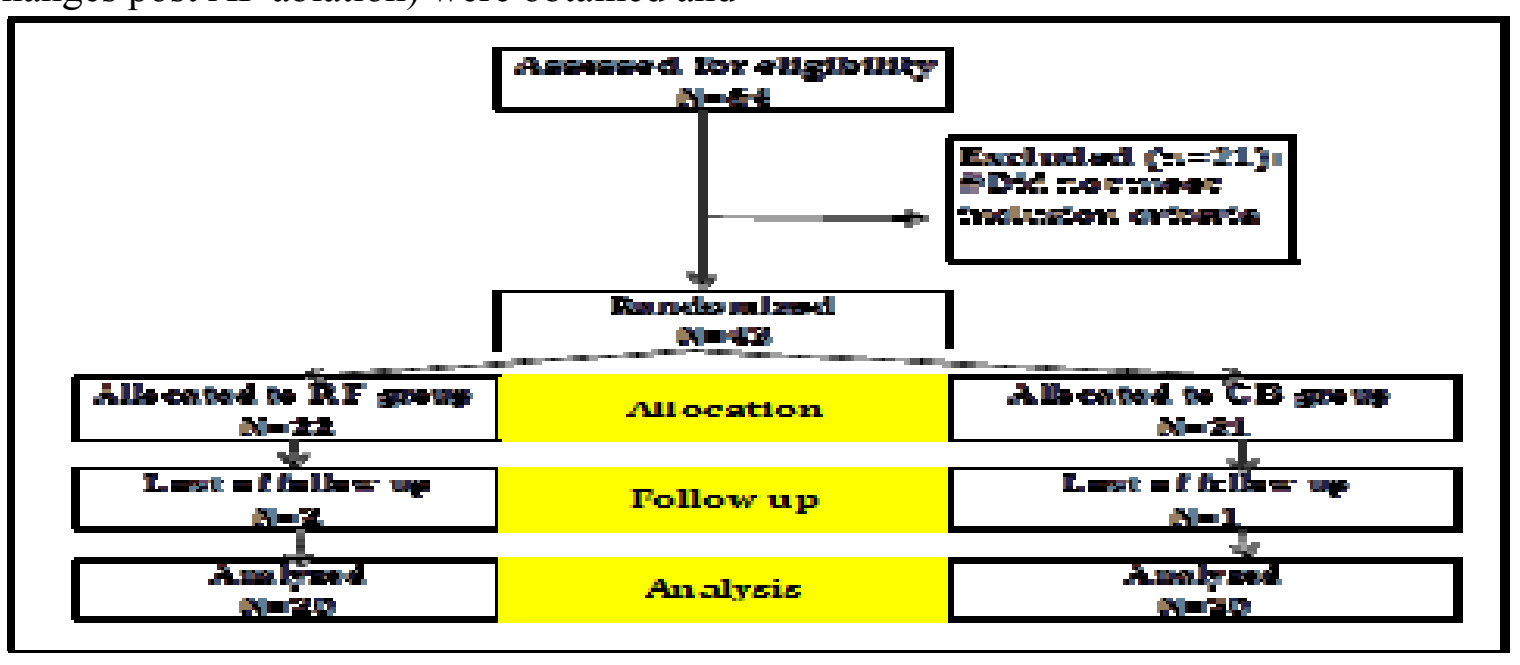

Figure 5): flow chart of the studied cases 


\section{Demographic data:}

In the RF group, the patients' ages ranged between 28 and 60 years old (mean age $43.7 \pm 11.3$ years), while in the $\mathrm{CB}$ group, the patients' ages ranged between 30 and 56 years old (mean age $43.3 \pm 8.9$ years). The percent of females among the RF group was $40 \%$, while in the CB group was

Table 1): Demographic characteristics and AF clinical characteristics among the studied groups

\begin{tabular}{|c|c|c|c|c|}
\hline \multicolumn{2}{|l|}{ Items } & $\mathrm{RF}(\mathrm{N}=20)$ & $\mathrm{CB}(\mathrm{N}=20)$ & $\mathrm{P}$ \\
\hline \multirow{2}{*}{$\begin{array}{l}\text { Age } \\
\text { (years) }\end{array}$} & Mean \pm SD & $43.7 \pm 11.3$ & $43.3 \pm 8.9$ & \multirow{2}{*}{${ }^{\wedge} 0.902$} \\
\hline & Range & $28.0-60.0$ & $30.0-56.0$ & \\
\hline \multirow{2}{*}{$\begin{array}{l}\text { BMI } \\
\left(\mathrm{kg} / \mathrm{m}^{2}\right)\end{array}$} & Mean \pm SD & $28.7 \pm 5.7$ & $29.4 \pm 6.1$ & \multirow{2}{*}{${ }^{\wedge} 0.708$} \\
\hline & Range & $19.0-40.0$ & $21.0-40.0$ & \\
\hline \multirow{2}{*}{ Sex } & Male & $12(60.0 \%)$ & $10(50.0 \%)$ & \multirow{2}{*}{$\# 0.525$} \\
\hline & Female & $8(40.0 \%)$ & $10(50.0 \%)$ & \\
\hline \multirow{2}{*}{ AF duration in months } & Median (IQR) & $\begin{array}{l}36.0 \\
(24.0-66.0)\end{array}$ & $\begin{array}{l}48.0 \\
(19.5-78.0)\end{array}$ & \multirow[t]{2}{*}{$\S 0.438$} \\
\hline & Range & $12.0-156.0$ & $12.0-240.0$ & \\
\hline \multirow{3}{*}{ AF frequency } & Daily & $4(20.0 \%)$ & $4(20.0 \%)$ & \multirow{3}{*}{$\& 0.492$} \\
\hline & Weekly & $15(75.0 \%)$ & $12(60.0 \%)$ & \\
\hline & Monthly & $1(5.0 \%)$ & $4(20.0 \%)$ & \\
\hline \multirow{2}{*}{ EHRA } & II & $11(55.0 \%)$ & $14(70.0 \%)$ & \multirow{2}{*}{$\# 0.327$} \\
\hline & III & $9(45.0 \%)$ & $6(30.0 \%)$ & \\
\hline \multirow{5}{*}{ 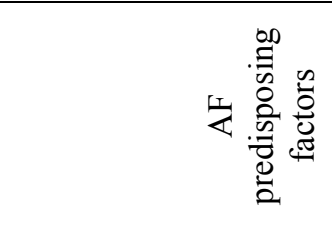 } & Idiopathic & $8(40.0 \%)$ & $9(45.0 \%)$ & $\# 0.749$ \\
\hline & HTN & $10(50.0 \%)$ & $8(40.0 \%)$ & $\# 0.525$ \\
\hline & $\mathrm{DM}$ & $4(20.0 \%)$ & $2(10.0 \%)$ & $\& 0.661$ \\
\hline & Smoker & $1(5.0 \%)$ & $3(15.0 \%)$ & $\& 0.605$ \\
\hline & Thyroid & $3(15.0 \%)$ & $0(0.0 \%)$ & $\& 0.231$ \\
\hline
\end{tabular}

IQR: Inter-quartile range. ${ }^{\wedge}$ Independent t-test, \#Chi square test. \&Fisher's Exact test. $\S$ Mann Whitney test. BMI: body mass index. EHRA: European Heart Rhythm Association grading of AF related symptoms

\section{Procedural details: Table (2)}

In both groups, PVI was attained by bilateral circumferential ablation surrounding ipsilateral pulmonary veins, and acute success was confirmed by both exit and entrance block. Brady-hypotensive response (vagal response) during ablation occurred in $60 \%$ of patients in both RF and CB groups equally. Additional cavo-tricuspid isthmus (CTI) ablation line was applied to 3 patients in the RF group. Left atrial scar was identified and ablated in 2 patients in the RF
$50 \%$. Statistical comparison between the two studied groups (radiofrequency and cryoballoon groups) regarding; Mean age in years, mean BMI in $\mathrm{kg} / \mathrm{m}^{2}$, gender, $\mathrm{AF}$ duration, $\mathrm{AF}$ frequency, EHRA class and $\mathrm{AF}$ predisposing factors revealed no significant statistical difference. Table (1) 
Wagdy A. Galal, et al.,

Table 2): Procedural details among the studied groups

\begin{tabular}{|c|c|c|c|c|}
\hline \multicolumn{2}{|l|}{ Items } & $\mathrm{RF}(\mathrm{N}=20)$ & $\mathrm{CB}(\mathrm{N}=20)$ & $\mathrm{P}$ \\
\hline \multicolumn{2}{|c|}{ Vagal response during ablation } & $12(60.0 \%)$ & $12(60.0 \%)$ & $\# 1.000$ \\
\hline \multicolumn{2}{|c|}{ Entrance block } & $20(100.0)$ & $20(100.0)$ & $\# 1.000$ \\
\hline \multicolumn{2}{|l|}{ Exit block } & $20(100.0)$ & $20(50.0 \%)$ & $\# 1.000$ \\
\hline \multirow{2}{*}{ Fluoro time (min) } & Mean \pm SD & $75.9 \pm 23.7$ & $36.7 \pm 8.0$ & \multirow{2}{*}{$\wedge<0.001 *$} \\
\hline & Range & $40.0-115.0$ & $24.0-46.0$ & \\
\hline \multirow{2}{*}{ procedure time (min) } & Mean \pm SD & $208.0 \pm 49.9$ & $135.5 \pm 49.1$ & \multirow{2}{*}{$\wedge<0.001 *$} \\
\hline & Range & $110.0-300.0$ & $90.0-240.0$ & \\
\hline \multicolumn{2}{|l|}{ PVI + CTI } & $3(15.0 \%)$ & -- & -- \\
\hline \multicolumn{2}{|l|}{ LA scar } & $2(10.0 \%)$ & -- & -- \\
\hline \multicolumn{2}{|l|}{ Smart touch catheter } & $9(45.0 \%)$ & -- & -- \\
\hline \multirow{2}{*}{ Lowest temp $\left(\mathrm{C}^{\mathrm{o}}\right)$} & Mean \pm SD & -- & $-48.8 \pm 4.1$ & \multirow[b]{2}{*}{--} \\
\hline & Range & -- & $-55.0--42.0$ & \\
\hline \multirow{2}{*}{ Extra cryo applications } & Median (IQR) & -- & $2.0(2.0-3.0)$ & \multirow[b]{2}{*}{--} \\
\hline & Range & -- & $0.0-4.0$ & \\
\hline
\end{tabular}

IQR: Inter-quartile range. ${ }^{\wedge}$ Independent t-test, \#Chi square test. \&Fisher's Exact test. §Mann Whitney test
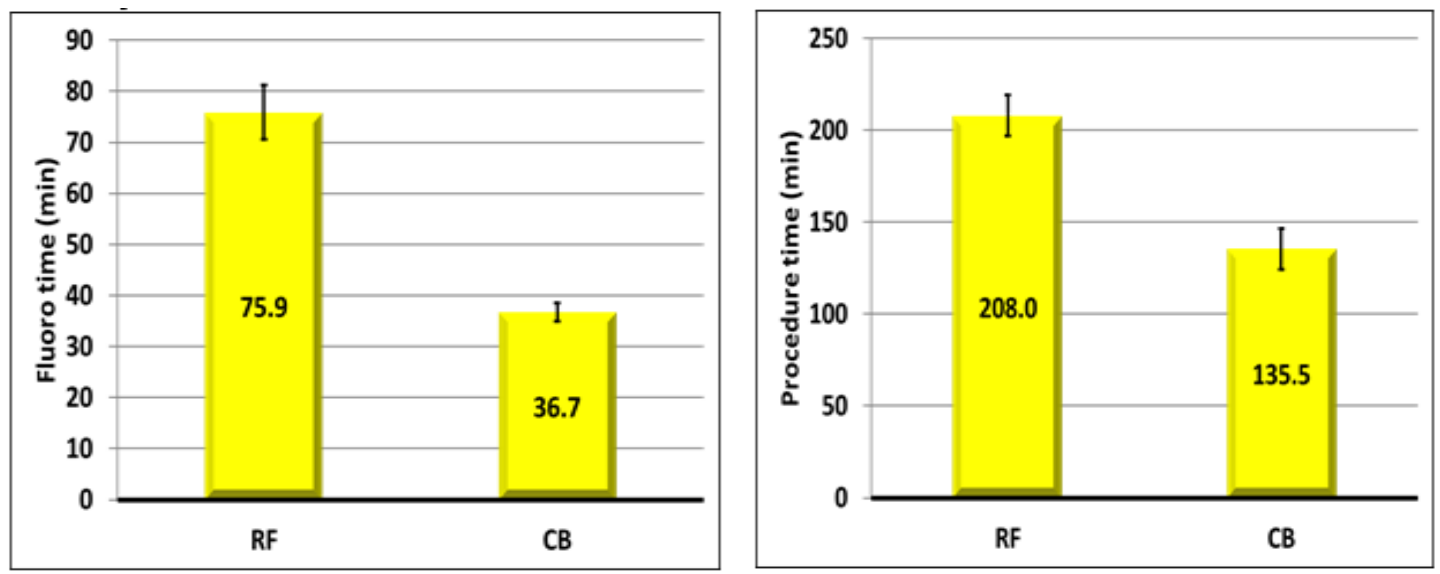

Figure 6): Fluoroscopy and Procedure time among the studied groups

\section{Follow up of echocardiographic parameters:}

No significant difference between the two studied groups regarding basal and month-12 change in LAD. LAD significantly decreased in RF group (Pvalue $=0.009)$ and non-significantly decreased in CB group. No significant difference between the two studied groups regarding basal and month-12 EF. EF significantly increased in RF group (Pvalue $=0.001)$ and non-significantly increased in CB group. (Figure 7) 

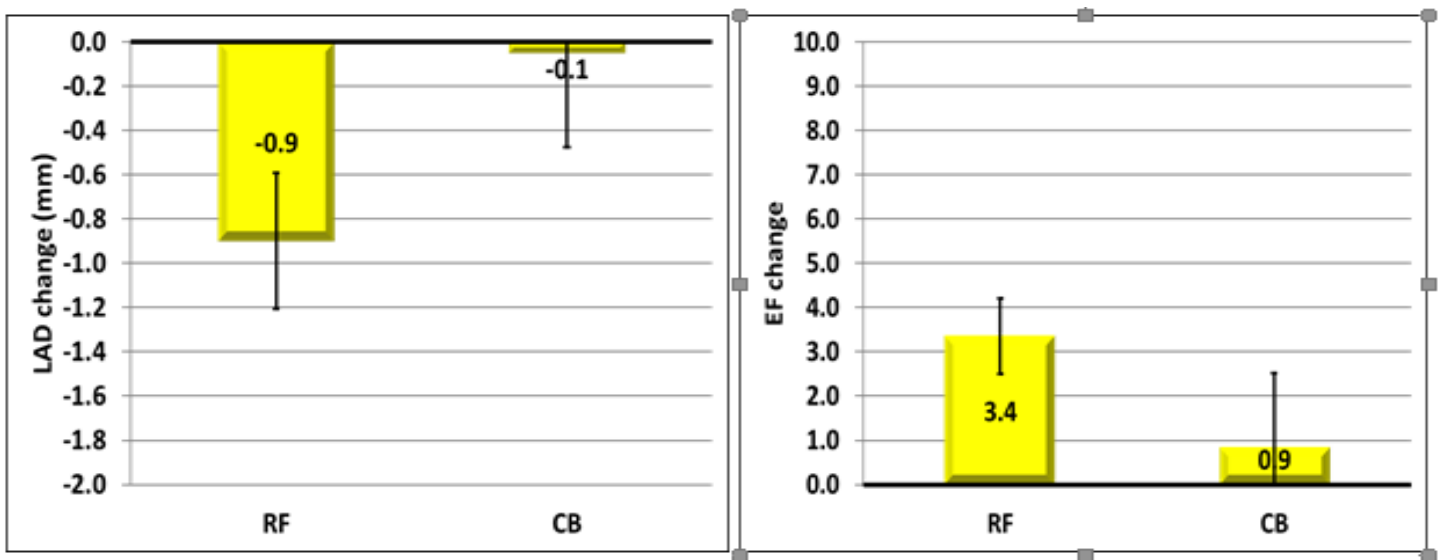

Figure 7): LAD and EF change after 12 months follow up

\section{Holter parameters (basal and follow up):}

Data was represented as basal parameters and delta change in the follow up parameters. The delta change was calculated by the difference between the basal value and the follow up value.

\subsection{Change in mean heart rate}

Table (3) shows that: there was no significant difference between the two studied groups regarding basal and the threepoint follow up of mean heart rate. The mean Heart rate increased in RF group more than $\mathrm{CB}$ group. However, this increase wasn't statistically significant. Figure (8)

Table 3): mean HR (beat/minute) among the studied groups

\begin{tabular}{|c|c|c|c|c|}
\hline \multicolumn{2}{|l|}{ Time } & $\mathrm{RF}(\mathrm{N}=20)$ & $\mathrm{CB}(\mathrm{N}=20)$ & $\wedge \mathrm{P}$ \\
\hline \multirow[t]{2}{*}{ Before } & Mean \pm SD & $78.0 \pm 12.6$ & $81.0 \pm 12.1$ & \multirow[t]{2}{*}{0.441} \\
\hline & Range & $57.0-100.0$ & $66.0-102.0$ & \\
\hline \multirow[t]{3}{*}{ Month-1 change } & Mean \pm SD & $4.1 \pm 12.0$ & $0.1 \pm 12.7$ & \multirow[t]{2}{*}{0.305} \\
\hline & Range & $-15.0-22.0$ & $-17.0-22.0$ & \\
\hline & $\# \mathrm{p}$ & 0.142 & 0.986 & \\
\hline \multirow[t]{3}{*}{ Month-3 change } & Mean \pm SD & $1.4 \pm 10.4$ & $0.4 \pm 7.4$ & \multirow[t]{2}{*}{0.715} \\
\hline & Range & $-15.0-20.0$ & $-12.0-13.0$ & \\
\hline & $\# \mathrm{p}$ & 0.555 & 0.834 & \\
\hline \multirow[t]{3}{*}{ Month-12 change } & Mean \pm SD & $3.2 \pm 12.5$ & $0.6 \pm 10.7$ & \multirow[t]{2}{*}{0.485} \\
\hline & Range & $-21.0-20.0$ & $-19.0-25.0$ & \\
\hline & $\# \mathrm{p}$ & 0.266 & 0.806 & \\
\hline
\end{tabular}

$\wedge$ Independent t-test, \#paired t-test (difference from basal). ${ }^{*}$ Signifcaint 
Wagdy A. Galal, et al.,

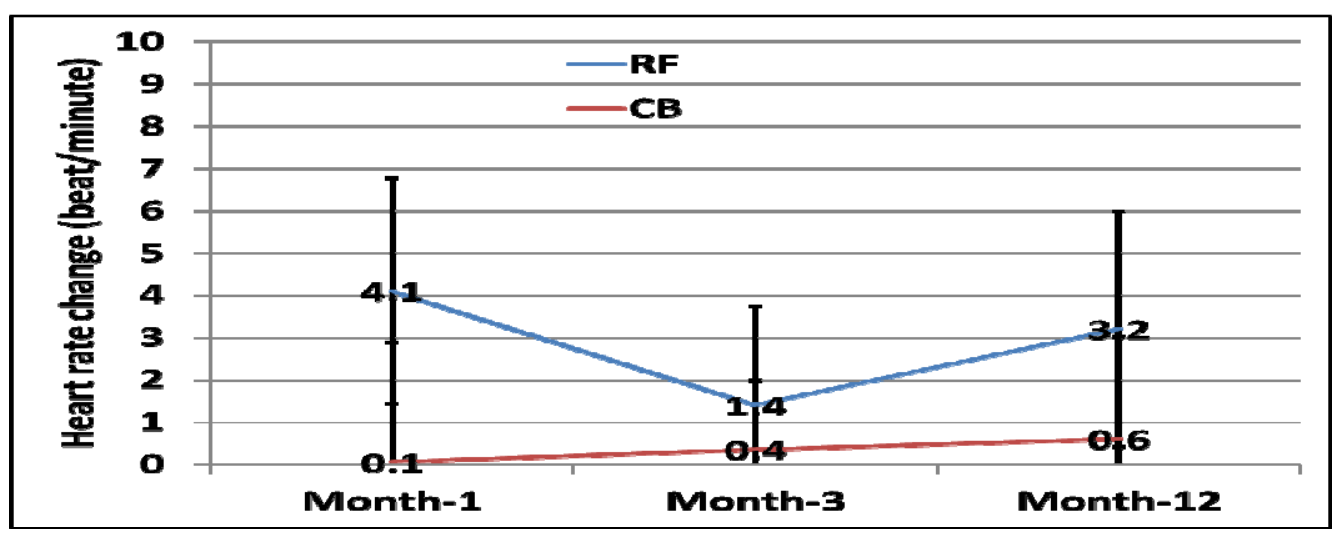

Figure 8): Mean heart rate change among the studied groups

\subsection{Change in SDNN}

Table (4) shows that: there was No significant difference between the two studied groups regarding basal and the threepoint follow up of SDNN. A high significant decrease in SDNN among the RF group in the three-point follow up with the maximum decrease at month-1 follow up. Furthermore, there was significant decrease in SDNN among the $\mathrm{CB}$ group in the three-point follow up with the maximum decrease at month-3 follow up. Figure (9)

Table 4): SDNN (msec) among the studied groups

\begin{tabular}{|c|c|c|c|c|}
\hline \multicolumn{2}{|l|}{ Time } & $\mathrm{RF}(\mathrm{N}=20)$ & $\mathrm{CB}(\mathrm{N}=20)$ & $\wedge \mathrm{P}$ \\
\hline \multirow[t]{2}{*}{ Before } & Mean \pm SD & $129.2 \pm 46.0$ & $139.6 \pm 36.0$ & \multirow[t]{2}{*}{0.428} \\
\hline & Range & $41.0-213.0$ & $90.0-210.0$ & \\
\hline \multirow[t]{3}{*}{ Month-1 change } & Mean \pm SD & $-50.3 \pm 47.1$ & $-30.3 \pm 48.4$ & \multirow[t]{2}{*}{0.192} \\
\hline & Range & $-137.0-36.0$ & $-68.0-97.0$ & \\
\hline & $\# \mathrm{p}$ & $<0.001 *$ & $0.012 *$ & \\
\hline \multirow[t]{3}{*}{ Month-3 change } & Mean \pm SD & $-38.1 \pm 39.8$ & $-38.1 \pm 41.8$ & \multirow[t]{2}{*}{1.000} \\
\hline & Range & $-136.0-20.0$ & $-143.0-9.0$ & \\
\hline & $\# \mathrm{p}$ & $<0.001 *$ & $0.001 *$ & \\
\hline \multirow[t]{3}{*}{ Month-12 change } & Mean \pm SD & $-28.8 \pm 47.4$ & $-33.0 \pm 40.8$ & \multirow[t]{2}{*}{0.766} \\
\hline & Range & $-138.0-36.0$ & $-140.0-10.0$ & \\
\hline & $\# \mathrm{p}$ & $0.014 *$ & $0.002 *$ & \\
\hline
\end{tabular}

${ }^{\wedge}$ Independent t-test, \#paired t-test (difference from basal). ${ }^{*}$ Signifcaint

\subsection{Change in rMSSD}

Table (5) shows that: there was No significant difference between the two studied groups regarding basal, month-1 and month-12 follow up of rMSSD. However, rMSSD significantly decreased at month-3 follow up in RF group more than the CB group (P-value 0.04). There was A significant decrease in rMSSD among the $\mathrm{RF}$ and $\mathrm{CB}$ groups in the three-point follow up with the maximum decrease at month-1 follow up in both groups. Figure (9) 
Table 5): rMSSD (mm) among the studied groups

\begin{tabular}{|c|c|c|c|c|}
\hline \multicolumn{2}{|l|}{ Time } & $\mathrm{RF}(\mathrm{N}=20)$ & $\mathrm{CB}(\mathrm{N}=20)$ & ${ }^{\wedge} \mathrm{P}$ \\
\hline \multirow[t]{2}{*}{ Before } & Mean \pm SD & $85.4 \pm 54.9$ & $71.8 \pm 38.0$ & \multirow[t]{2}{*}{0.367} \\
\hline & Range & $24.0-221.0$ & $17.0-143.0$ & \\
\hline \multirow[t]{3}{*}{ Month-1 change } & Mean \pm SD & $-56.1 \pm 64.4$ & $-27.1 \pm 23.0$ & \multirow[t]{2}{*}{0.070} \\
\hline & Range & $-208.0-53.0$ & $-83.0-1.0$ & \\
\hline & $\# p$ & $0.001 *$ & $<0.001^{*}$ & \\
\hline \multirow[t]{3}{*}{ Month-3 change } & Mean \pm SD & $-54.6 \pm 60.6$ & $-21.6 \pm 32.0$ & \multirow[t]{2}{*}{$0.040^{*}$} \\
\hline & Range & $-210.0-21.0$ & $-104.0-14.0$ & \\
\hline & $\# \mathrm{p}$ & $0.001 *$ & $0.007 *$ & \\
\hline \multirow[t]{3}{*}{ Month-12 change } & Mean \pm SD & $-44.8 \pm 56.7$ & $-19.8 \pm 36.1$ & \multirow[t]{2}{*}{0.104} \\
\hline & Range & $-209.0-20.0$ & $-95.0-37.0$ & \\
\hline & $\# \mathrm{p}$ & $0.002 *$ & $0.024 *$ & \\
\hline
\end{tabular}

${ }^{\wedge}$ Independent t-test, \#paired t-test (difference from basal). ${ }^{*}$ Signifcaint
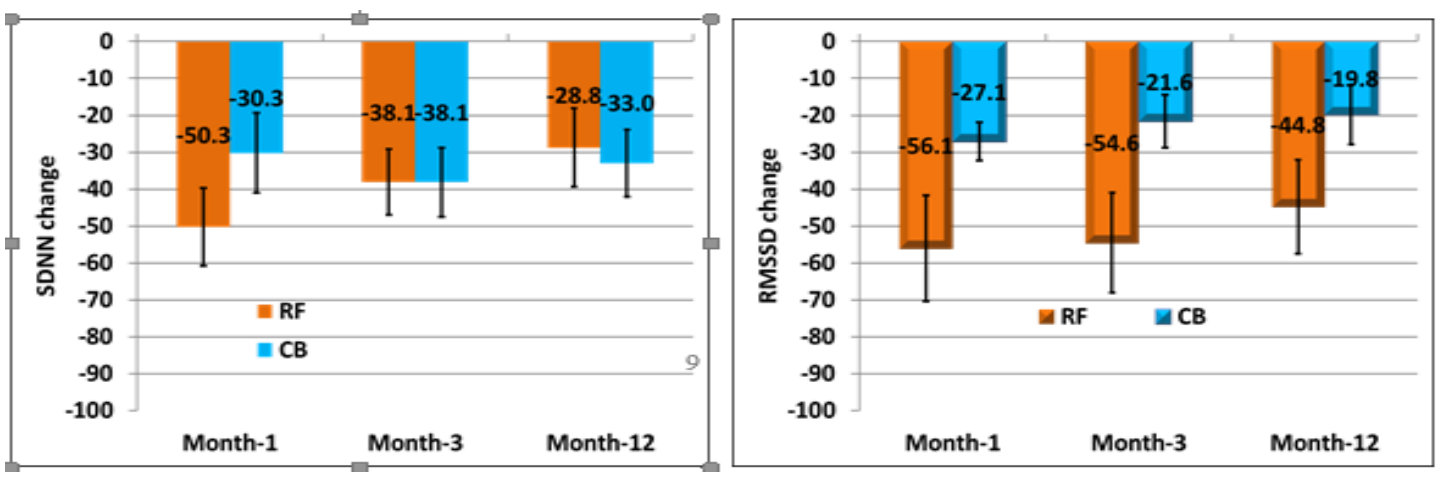

Figure 9): SDNN change among the studied groups

\subsection{Triangular index}

Table (6) shows that: There was no significant difference between the studied groups regarding basal and the three-point index was significantly decreased in RF group at months- 1 and month-3 follow up and significantly decreased in $\mathrm{CB}$ group only at month-1 follow up.

follow up of triangular index. Triangular

Table 6): Triangular index among the studied groups

\begin{tabular}{|c|c|c|c|c|}
\hline \multicolumn{2}{|l|}{ Time } & $\mathrm{RF}(\mathrm{N}=20)$ & $\mathrm{CB}(\mathrm{N}=20)$ & $\wedge^{\wedge} \mathrm{P}$ \\
\hline \multirow{2}{*}{ Before } & Mean \pm SD & $35.0 \pm 11.1$ & $33.0 \pm 9.9$ & \multirow[t]{2}{*}{0.551} \\
\hline & Range & $20.0-55.0$ & $16.0-45.0$ & \\
\hline \multirow[t]{3}{*}{ Month-1 change } & Mean \pm SD & $-9.3 \pm 9.8$ & $-9.1 \pm 11.3$ & \multirow[t]{2}{*}{0.941} \\
\hline & Range & $-32.0-15.0$ & $-28.0-5.0$ & \\
\hline & $\# \mathrm{p}$ & $<0.001^{*}$ & $0.002 *$ & \\
\hline \multirow[t]{3}{*}{ Month-3 change } & Mean \pm SD & $-7.2 \pm 14.2$ & $-4.2 \pm 11.0$ & \multirow[t]{2}{*}{0.467} \\
\hline & Range & $-38.0-10.0$ & $-21.0-8.0$ & \\
\hline & $\# \mathrm{p}$ & $0.036^{*}$ & 0.105 & \\
\hline \multirow[t]{3}{*}{ Month-12 change } & Mean \pm SD & $-2.6 \pm 12.4$ & $-2.9 \pm 7.0$ & \multirow[t]{2}{*}{0.926} \\
\hline & Range & $-36.0-24.0$ & $-13.0-5.0$ & \\
\hline & $\# \mathrm{p}$ & 0.370 & 0.086 & \\
\hline
\end{tabular}

$\wedge$ Independent t-test, \#paired t-test (difference from basal). *Signifcaint 


\subsection{Change in $\mathrm{LF} / \mathrm{HF}$ ratio}

Table (7) shows that: There was no significant difference between the studied groups regarding basal LF/HF. LF/HF elevation was higher in RF group, but the differences between both groups were significant only at month-3. LF/HF was significantly increased in RF group in the three-point follow up. However, significantly increased in $\mathrm{CB}$ group only at month-1 follow up.

Table 7): Change in LF/HF ratio among the studied groups

\begin{tabular}{|c|c|c|c|c|}
\hline \multicolumn{2}{|l|}{ Time } & $\mathrm{RF}(\mathrm{N}=20)$ & $\mathrm{CB}(\mathrm{N}=20)$ & $\wedge \mathrm{P}$ \\
\hline \multirow[t]{2}{*}{ Before } & Mean \pm SD & $1.46 \pm 0.73$ & $1.44 \pm 0.50$ & \multirow[t]{2}{*}{0.920} \\
\hline & Range & $0.50-3.20$ & $0.90-2.60$ & \\
\hline \multirow[t]{3}{*}{ Month-1 change } & Mean \pm SD & $1.07 \pm 1.38$ & $1.01 \pm 1.82$ & \multirow[t]{2}{*}{0.915} \\
\hline & Range & $-1.00-3.80$ & $-1.10-5.30$ & \\
\hline & $\# \mathrm{p}$ & $0.003 *$ & $0.023^{*}$ & \\
\hline \multirow[t]{3}{*}{ Month-3 change } & Mean \pm SD & $1.70 \pm 2.60$ & $0.26 \pm 0.58$ & \multirow[t]{2}{*}{$0.025^{*}$} \\
\hline & Range & $-1.30-7.40$ & $-0.70-1.50$ & \\
\hline & $\# \mathrm{p}$ & $0.009^{*}$ & 0.065 & \\
\hline \multirow[t]{3}{*}{ Month-12 change } & Mean \pm SD & $0.41 \pm 0.80$ & $0.03 \pm 0.46$ & \multirow[t]{2}{*}{0.077} \\
\hline & Range & $-1.40-2.40$ & $-0.80-1.00$ & \\
\hline & $\# p$ & $0.035^{*}$ & 0.775 & \\
\hline
\end{tabular}

${ }^{\wedge}$ Independent t-test, \#paired t-test (difference from basal). *Signifcaint

\section{Orthostatic hypotension}

Table (8) shows that: There was No significant difference between the studied groups regarding orthostatic hypotension before AF ablation and at the three-point follow up.

Table 8): Orthostatic hypotension among the studied groups

\begin{tabular}{|l|l|l|l|}
\hline Items & $\mathrm{RF}(\mathrm{N}=20)$ & $\mathrm{CB}(\mathrm{N}=20)$ & $\& \mathrm{P}$ \\
\hline Before & $0(0.0 \%)$ & $2(10.0 \%)$ & 0.487 \\
\hline Month-1 & $3(15.0 \%)$ & $6(30.0 \%)$ & 0.451 \\
\hline Month-3 & $3(15.0 \%)$ & $6(30.0 \%)$ & 0.451 \\
\hline Month-12 & $0(0.0 \%)$ & $2(10.0 \%)$ & 0.487 \\
\hline \#P & 1.000 & 1.000 & \\
\hline
\end{tabular}

\&Fisher's Exact test. \#McNemr test

\section{AF recurrence}

Table (9) and figure (10) shows that: AF recurrence in the blanking period was less frequent in RF group (5 patients, $25.0 \%$ ) Vs CB group (9 patients, $45.0 \%$ ). However, the difference wasn't statistically significant. Four patients within the RF and $\mathrm{CB}$ groups experienced clinical recurrence after 3 months follow up. Additionally, only one patient in RF group and 3 patients in $\mathrm{CB}$ group had clinical recurrence after 3 months till 12 months follow up. By 12 months follow up, Total 5/20 in RF group and 7/20 in $\mathrm{CB}$ had recurrence (success rate in $\mathrm{RF}$ group $75 \%$ and in $\mathrm{CB}$ group 65\%). $\mathrm{AF}$ recurrence within 12 months follow up was less frequent in RF group (5 patients, $25.0 \%$ ) Vs CB group (7 patients, 35.0\%). However, the difference wasn't statistically significant. 
Table 9): AF recurrence among the studied groups

\begin{tabular}{|l|l|l|l|}
\hline Items & $\mathrm{RF}(\mathrm{N}=20)$ & $\mathrm{CB}(\mathrm{N}=20)$ & $\mathrm{P}$ \\
\hline Month-1 (blanking) & $5(25.0 \%)$ & $9(45.0 \%)$ & $\# 0.185$ \\
\hline Month-3 (clinical) & $4(20.0 \%)$ & $4(20.0 \%)$ & $\& 1.000$ \\
\hline Month-12 (clinical) & $5(25.0 \%)$ & $7(35.0 \%)$ & $\# 0.490$ \\
\hline
\end{tabular}

\#Chi square test. \&Fisher's Exact test.

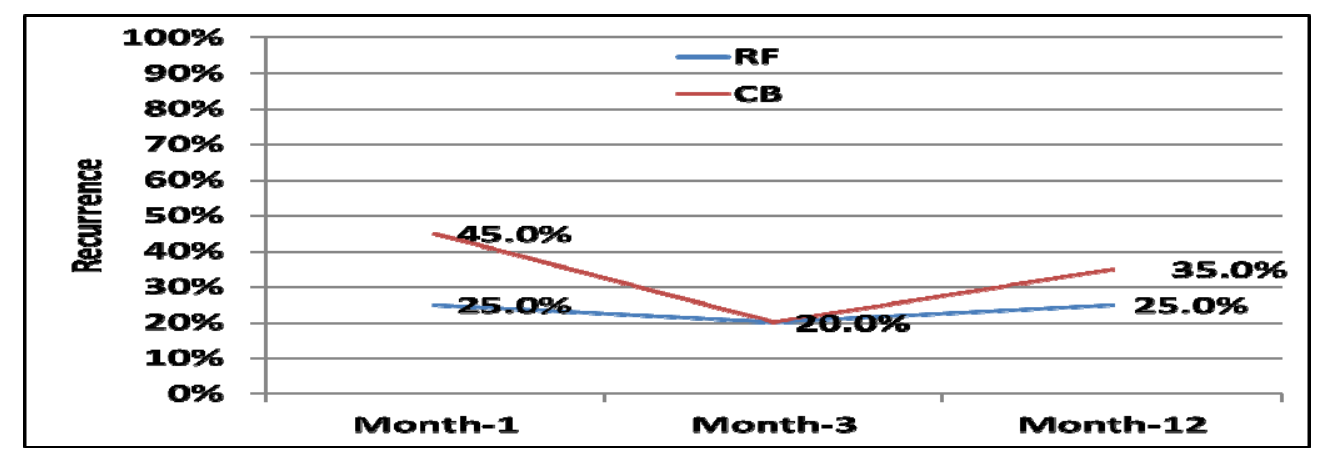

Figure 10): AF recurrence among the studied groups

7. Periprocedural complications:

Table (10) shows that: There was No significant difference between the studied groups regarding periprocedural complications.

Eight patients $(40.0 \%)$ among the $\mathrm{RF}$ group Vs 12 patients $(60.0 \%)$ among the CB group complained of early post ablation palpitations due to high sinus rate $\geq 90 \mathrm{bpm}$ not related AF recurrence which was comparable. There was no statistically significant difference between the two studied groups regarding post procedural complications; 3 cases in RF group Vs 2 cases in $\mathrm{CB}$ group.

Table (10): Periprocedural complications among the studied groups

\begin{tabular}{|l|l|l|l|}
\hline ITEMS & $\begin{array}{l}\mathrm{RF} \\
\mathrm{N}=20)\end{array}$ & $\begin{array}{l}\mathrm{CB} \\
(\mathrm{N}=20)\end{array}$ & $\mathrm{P}$ \\
\hline Post ablation high sinus rate $\geq 90 \mathrm{bpm}$ & $8(40.0 \%)$ & $12(60.0 \%)$ & $\# 0.206$ \\
\hline Chest infection & $2(10.0 \%)$ & $0(0.0 \%)$ & $\# 0.487$ \\
\hline Coronary events & $0(0.0 \%)$ & $1(5.0 \%)$ & $\& 1.000$ \\
\hline Pericardial effusion & $0(0.0 \%)$ & $1(5.0 \%)$ & $\& 1.000$ \\
\hline Gastric hypomotility & $1(5.0 \%)$ & $0(0.0 \%)$ & $\& 1.000$ \\
\hline Phrenic palsy & $0(0.0 \%)$ & $0(0.0 \%)$ & -- \\
\hline
\end{tabular}

\#Chi square test. \&Fisher's Exact test.

\section{DISCUSSION}

This study looked at the incidence and the value of post AF ablation cardiac autonomic dysfunction while comparing between radiofrequency and cryoballoon ablation techniques and the main findings were as follows:
- No significant difference between radiofrequency (RF) and cryoballoon (CB) groups regarding demographic data, AF predisposing factors and clinical characteristics.

- Cryoballoon ablation was comparable to RF ablation regarding efficacy. The 
efficacy data in the form of freedom from $\mathrm{AF}$ at one year using radiofrequency technique was slightly higher than cryoballoon technique $(75 \%$ Vs $65 \%$ respectively). However, this difference wasn't statistically significant.

- The incidence of complications was comparable between the two studied groups (3 cases in RF group Vs 2 cases in $\mathrm{CB}$ group).

- The cryoballoon procedure had less procedural time and fluoroscopy time than the radiofrequency procedure.

- During the 3-point follow up of HRV parameters (month-1, month-3 and month-12), changes in time domain and frequency domain parameters persisted for 12 months after both techniques, being more pronounced in $\mathrm{RF}$ group than $\mathrm{CB}$ group.

In this study, 40 patients were studied, with a mean age of $43.7 \pm 11.3$ years for the RF group and $43.3 \pm 8.9$ for the CB group and females representing $40 \quad \% \quad$ vs $50 \%$ respectively. All patients were suffering from paroxysmal atrial fibrillation and underwent a de novo ablation procedure.

Due to the one-shot nature of energy delivery in the cryoballoon, the procedural time tends to be shorter. Additionally, the freeze cycle duration and delivery of a bonus freeze influence the duration of the procedure with new shorter application times associated with faster procedures ${ }^{(14)}$. In the current study, mean procedural and fluoroscopy times were significantly less in the $\mathrm{CB}$ group compared to the $\mathrm{RF}$ group (procedural time in 135.5 vs. 208 minutes, $\mathrm{p}<0.0001$ ) and (fluoroscopy time 75.9 vs. 36.7 minutes, $\mathrm{p}<0.0001)$ in $\mathrm{CB}$ and $\mathrm{RF}$ groups respectively. Results by Luik et al., also demonstrated lower mean procedural time for the cryoballoon (161 vs. 174 minutes, $\mathrm{p}=0.006$ ) while mean fluoroscopy time was equivalent (24.5 vs. 24 minutes, $\mathrm{p}=0.632) .{ }^{(15)}$ The briefer procedural times in the current study cryoballoon group can be explained by the use of the secondgeneration balloon only, which is more efficient at energy delivery. ${ }^{(16)}$

In the current study, the efficacy data in the form of freedom from $\mathrm{AF}$ at one year was $65 \%$ for $\mathrm{CB}$ vs $75 \%$ for RF ablation (pvalue 0.49 ). These findings were comparable with results of the recent randomised controlled trial conducted by Kuck et al. where non inferiority of cryoballoon to radiofrequency ablation was proved at 12 months follow up (1-year event-rate estimates, $34.6 \%$ and $35.9 \%$, respectively; $\mathrm{HR}, 0.96 ; 95 \% \mathrm{CI}, 0.76$ to $1.22 ; \mathrm{p}<0.001$ for noninferiority) $\stackrel{(17)}{ }$.

After Coumel et al. $\stackrel{(2)}{\text { demonstrated }}$ significant autonomic in-fluences in AF, numerous studies have shown relationships between the cardiac autonomic function and AF. ${ }^{(1,7,18-21)}$ Insufficient autonomic modulation determined by HRV parameters could be associated with $\mathrm{AF}$ recurrence. ${ }^{(21,22)}$

This study evaluated long-term HRV changes (time domain and frequency domain parameters) after radiofrequency and cryoballoon AF ablation and demonstrated that most of HRV changes persisted for 1 year after both techniques. Pappone et al.,(1) demonstrated that reductions in HRV parameters persisted for 3 months and recovered at 6 months after RF ablation. These effects of autonomic denervation were not maintained long term due to using conventional non irrigation tip catheters. Similar to the current study Bauer et al. (21) and Kang et al. ${ }^{(20)}$ found that persistent cardiac autonomic dysfunction (depressed HRV parameters) for 1 year was associated with circumferential pulmonary vein ablation more than segmental pulmonary vein ablation and with open irrigation tip catheters (OITC) more than conventional catheters. The mechanism of these improvements might be that the OITC increases transmural lesion formation and 
epicardial denervation more than the conventional catheter does.

In the current study SDNN showed a highly significant decrease in the 3-point follow up among the RF group and a significant decrease in the 3-point follow up among the $\mathrm{CB}$ group. However, there was no statistically significant difference between the two techniques at any follow up point. The same changes were noticed regarding rMSSD. However, it decreased significantly in 3-month follow up in RF group more than $\mathrm{CB}$ group. Pappone et al., (1) demonstrated a significant decrease in SDNN and rMSSD which were maximally depressed at 1 month follow up, persisted for 3 months follow up and these results were comparable to the current study. However, these changes normalized to basal levels by 6 months. While in the current study, the decrease in SDNN and rMSSD persisted for 1 year follow up. This difference may be explained by using non irrigation tip catheters in their study. Kang et al. ${ }^{(20)}$ demonstrated a significant decrease in rMSSD in 3-month and 12-month follow up which was similar to the current study. Furthermore, they revealed a strong correlation between this persistent decrease in rMSSD and long-term success (AF free for mean 22 months \pm 8 ). However, they didn't use SDNN in their study. Regarding HRV changes post cryoballoon ablation, Yanagisawa et al. ${ }^{(23)}$ followed rMSSD and SDNN in 41 cryoballoon ablated patients and demonstrated a significant decrease in both parameters within 1 month follow up, which was comparable to the $\mathrm{CB}$ group in the current study, except for shorter period of follow up in their study.

In this study, LF/HF persistently increased in RF group through the 3-point follow up but not the CB group (Only significant increase in LF/HF in month-1 follow up). So, HRV changes, especially frequency domain parameters. were more pronounced in the RF group more than the
CB group. Pappone et al., ${ }^{(1)}$ demonstrated a significant increase in LF/HF which was maximally elevated at 1 month follow up, persisted for 3 months follow up and these results were comparable to the current study. However, these changes normalized to basal levels by 6 months. While in the current study, the increase in LF/HF persisted for 1 year follow up in the RF group. This difference may be explained by using non irrigation tip catheters in their study. Kang et al. ${ }^{(20)}$ demonstrated a significant increase in LF/HF in 3-month and 12-month follow up which was similar to the current study. Furthermore, they revealed a strong correlation between the decrease in $\mathrm{LF} / \mathrm{HF}>0.27$ and clinical recurrence. In other words, the persistent increase in LF/HF (vagal denervation or sympathetic dominance) had less AF clinical recurrence.

In the current study Mean HR increased in the 3-point follow up and mean RR decreased in the 3-point follow up among both groups being more pronounced in RF group more than CB group. However, these changes weren't statistically significant. Pappone et al., (1) demonstrated a significant increase in mean HR which was maximally elevated at 1 month follow up, persisted for 3 months follow up and resolved 6 months later. The non-significant elevation in HR in the current study may be explained by concomitant use of betablockers in some patients with symptomatic post $\mathrm{AF}$ ablation high sinus rate.

Autonomic fluctuations are correlated with AF initiation and potentially maintenance. ${ }^{(24)}$ The current study evaluated orthostatic hypotension pre and post $\mathrm{AF}$ ablation as a sign of autonomic dysfunction. Only 2 patients among the $\mathrm{CB}$ group had basal $\mathrm{OH}$. For 1 year follow up additional 3 patients in RF group and 4 patients in $\mathrm{CB}$ group developed orthostatic hypotension at month-1 and month-3 follow up. These changes resolved by month-12. However, there was no statistically significant 
difference regarding $\mathrm{OH}$ between $\mathrm{RF}$ and $\mathrm{CB}$ groups in basal and 3-point follow up. In the current study only one patient $(5 \%)$ in the RF group developed gastrointestinal symptoms in the form of dyspepsia and occasional vomiting which spontaneously resolved after 3 months with symptomatic treatment. Kuck et al., In the FIRE and ICE trial ${ }^{(17)}$ reported 2 patients $(0.5 \%)$ in $\mathrm{RF}$ group and 1 patient $(0.3 \%)$ in the $\mathrm{CB}$ group with gastric hypomotility. Their results are comparable with the current study.

In the current study, follow up of left ventricular systolic function (EF) and left atrium anteroposterior diameter (LAD) one year after the procedure revealed an increase in mean $\mathrm{EF}(\%)$ in both groups $(+3.4$ in $\mathrm{RF}$ and +0.9 in $\mathrm{CB}$ ) and a decrease in mean LAD (mm) in both groups (-0.9 in RF and 0.1 in $\mathrm{CB}$ ), being more pronounced in the $\mathrm{RF}$ group. So, vagal denervation and persistent change in HRV parameters wasn't associated with deterioration in LV systolic function or LA remodeling. Similarly, Yu HT et al., (13) demonstrated that post AF ablation high sinus rate and vagal denervation wasn't associated with adverse cardiac effects demonstrated by 1-year follow up echocardiogram.

\section{LIMITATIONS OF THE STUDY:}

On interpretation of the study results, certain limitations should be in mind. First, this study was relatively small, where consecutive patients were recruited after randomization. Results will need to be confirmed in a larger randomised study to better evaluate differences between groups and assess the impact of HRV changes on clinical outcome. Another limitation of our study is that, only de-novo AF ablation patients were included in this study. Heart rate variability changes and vagal denervation needs to be studied in redo AF ablation procedures. Another point was that the left atrial size was represented by the anteroposterior diameter which may be misleading. The assessment would be better made using indexed left atrial volume, or assessment of volume by cardiac magnetic resonance.

\section{Conclusion:}

Heart rate variability changes persisted for 12 months after AF ablation, being more pronounced in radiofrequency group than cryoballoon group and wasn't associated with adverse cardiac events. Cryoballoon based pulmonary vein isolation is an effective alternative to magnetic navigationguided radiofrequency ablation in patients suffering from symptomatic drug refractory paroxysmal atrial fibrillation. The cryoballoon procedure is safe with shorter procedural times.

\section{REFERENCE:}

1. Pappone C, Santinelli V, Manguso F, Vicedomini G, Gugliotta F, Augello G, Et Al. Pulmonary Vein Denervation Enhances Long-Term Benefit After Circumferential Ablation For Paroxysmal Atrial Fibrillation. Circulation. 2004;109(3):327-34.

2. Coumel P. Autonomic Influences In Atrial Tachyarrhythmias. Journal Of Cardiovascular Electrophysiology. 1996;7(10):999-1007.

3. Elvan A, Pride HP, Eble JN, Zipes DP. Radiofrequency Catheter Ablation Of The Atria Reduces Inducibility And Duration Of Atrial Fibrillation In Dogs. Circulation. 1995;91(8):2235-44.

4. Chiou C-W, Zipes DP. Selective Vagal Denervation Of The Atria Eliminates Heart Rate Variability And Baroreflex Sensitivity While Preserving Ventricular Innervation. Circulation. 1998;98(4):360-8.

5. Voss A, Schulz S, Schroeder R, Baumert M, Caminal P. Methods Derived From Nonlinear Dynamics For Analysing Heart Rate Variability. Philosophical Transactions Of The Royal Society Of London A: Mathematical, Physical And Engineering Sciences. 2009;367(1887):277-96. 
6. Soejima K, Akaishi M, Mitamura H, Ogawa S, Sakurada H, Okazaki H, Et Al. Increase In Heart Rate After Radiofrequency Catheter Ablation Is Mediated By Parasympathetic Nervous Withdrawal And Related To Site Of Ablation. Journal Of Electrocardiology. 1997;30(3):239-46.

7. Hsieh M-H, Chiou C-W, Wen Z-C, Wu C-H, Tai C-T, Tsai C-F, Et Al. Alterations Of Heart Rate Variability After Radiofrequency Catheter Ablation Of Focal Atrial Fibrillation Originating From Pulmonary Veins. Circulation. 1999;100(22):2237-43.

8. Kang K-W, Pak H-N, Park J, Park JG, Uhm JS, Joung B, Et Al. Additional Linear Ablation From The Superior Vena Cava To Right Atrial Septum After Pulmonary Vein Isolation Improves The Clinical Outcome In Patients With Paroxysmal Atrial Fibrillation: Prospective Randomized Study. Europace. 2014;16(12):1738-45.

9. Chiou C-W, Eble JN, Zipes DP. Efferent Vagal Innervation Of The Canine Atria And Sinus And Atrioventricular Nodes: The Third Fat Pad. Circulation. 1997;95(11):2573-84.

10. Schatz I, Bannister R, Freeman R, Jankovic J, Koller W, Low PA, Et Al. Consensus Statement On The Definition Of Orthostatic Hypotension, Pure Autonomic Failure And Multiple System Atrophy. Clinical Autonomic Research. 1996;6(2):125-6.

11. Tzeis S, Pastromas S, Sikiotis A, Andrikopoulos G. Cryoablation In Persistent Atrial Fibrillation - A Critical Appraisal. Netherlands Heart Journal : Monthly Journal Of The Netherlands Society Of Cardiology And The Netherlands Heart Foundation. 2016;24(9):498-507.

12. Electrophysiology Tfotesoctnasop. Heart Rate Variability: Standards Of Measurement, Physiological Interpretation, And Clinical Use. Circulation. 1996;93(5):1043-65.
13. Yu HT, Kim TH, Uhm JS, Kim JY, Joung B, Lee MH, Et Al. Prognosis Of High Sinus Heart Rate After Catheter Ablation For Atrial Fibrillation. Europace. 2017;19(7):1132-9.

14. Straube F, Dorwarth U, Hartl S, Bunz B, Wankerl M, Ebersberger U, Et Al. Outcome Of Paroxysmal Atrial Fibrillation Ablation With The Cryoballoon Using Two Different Application Times: The 4- Versus 3-Min Protocol. Journal Of Interventional Cardiac Electrophysiology : An International Journal Of Arrhythmias And Pacing. 2016;45(2):169-77.

15. Luik A, Radzewitz A, Kieser M, Walter M, Bramlage P, Hörmann P, Et Al. Cryoballoon Versus Open Irrigated Radiofrequency Ablation In Patients With Paroxysmal Atrial Fibrillation. Circulation. 2015;132(14):1311-9.

16. Aryana A, Morkoch S, Bailey S, Lim HW, Sara R, d'Avila A, Et Al. Acute Procedural And Cryoballoon Characteristics From Cryoablation Of Atrial Fibrillation Using The First- And Second-Generation Cryoballoon: A Retrospective Comparative Study With Follow-Up Outcomes. Journal Of Interventional Cardiac Electrophysiology : An International Journal Of Arrhythmias And Pacing. 2014;41(2):177-86.

17. Kuck K-H, Brugada J, Fürnkranz A, Metzner A, Ouyang F, Chun KRJ, Et Al. Cryoballoon Or Radiofrequency Ablation For Paroxysmal Atrial Fibrillation. New England Journal Of Medicine. 2016;374(23):2235-45.

18. Armour JA, Murphy DA, Yuan BX, Macdonald S, Hopkins DA. Gross And Microscopic Anatomy Of The Human Intrinsic Cardiac Nervous System. The Anatomical Record: An Official Publication Of The American Association Of Anatomists. 1997;247(2):289-98.

19. Choi E-K, Shen MJ, Han S, Kim D, Hwang S, Sayfo S, Et Al. Intrinsic Cardiac Nerve Activity And Paroxysmal Atrial Tachyarrhythmia In Ambulatory Dogs. Circulation. 2010;121(24):2615. 
20. KANG KW, Kim TH, Park J, Uhm JS, Joung B, Hwang C, Et Al. Long-Term Changes In Heart Rate Variability After Radiofrequency Catheter Ablation For Atrial Fibrillation: 1-Year Follow-Up Study With Irrigation Tip Catheter. Journal Of Cardiovascular Electrophysiology. 2014;25(7):693-700.

21. Bauer A, Deisenhofer I, Schneider R, Zrenner B, Barthel P, Karch M, Et Al. Effects Of Circumferential Or Segmental Pulmonary Vein Ablation For Paroxysmal Atrial Fibrillation On Cardiac Autonomic Function. Heart Rhythm. 2006;3(12):142835.

22. Yamada T, Yoshida N, Murakami Y, Okada T, Yoshida Y, Muto M, Et Al. Vagal Modification Can Be A Valid Predictor Of Late Recurrence Of
Paroxysmal Atrial Fibrillation Independent Of The Pulmonary Vein Isolation Technique. Circulation Journal. 2009;73(9):1606-11.

23. Yanagisawa S, Inden $\mathbf{Y}$, Mizutani $\mathbf{Y}$, Fujii A, Kamikubo Y, Kanzaki Y, Et Al. Vagal Response In Cryoballoon Ablation Of Atrial Fibrillation And Autonomic Nervous System: Utility Of Epicardial Adipose Tissue Location. Journal Of Arrhythmia. 2017;33(4):275-82.

24. Agarwal SK, Alonso A, Whelton SP, Soliman EZ, Rose KM, Chamberlain AM, Et Al. Orthostatic Change In Blood Pressure And Incidence Of Atrial Fibrillation: Results From A Bi-Ethnic Population Based Study. Plos One. 2013;8(11):E79030. 


\section{تقييم خلل الوظائف التلقائية للقلب بعد عزل الأوردة الرئوية وتأثير ها على نجاح كي الذبذبة الأذينية الانتيابية}

وجدي جلال , محمد أمين , .لمياء علام , محمد موسى الئ

مقدمة: الذبذبة الأذينية هي أكثر أنواع عدم انتظام ضربات القلب انتشار اوتمثل قضية صحية عامة رئيسية. في الآونة

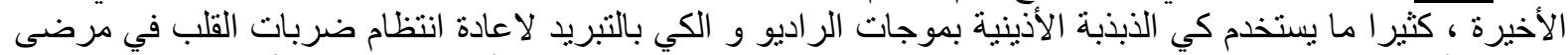

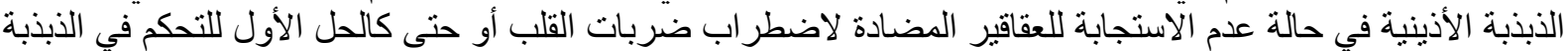

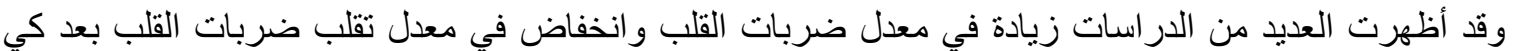

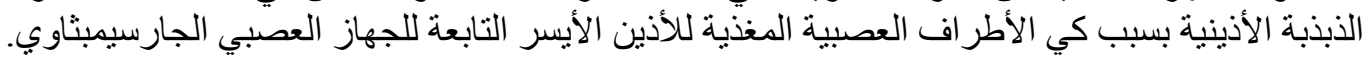

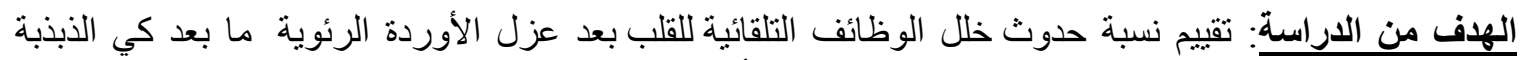

الأذينية و تقييم القيمة العملية لحدوث هذا الخلل. وذلك بعد عزل الأوردة الرئوية باستخدام موجات الترة الر اديو و الكي بالتبريد.

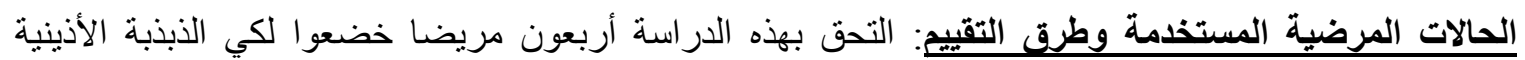

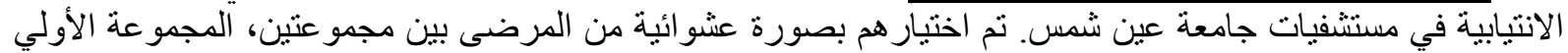

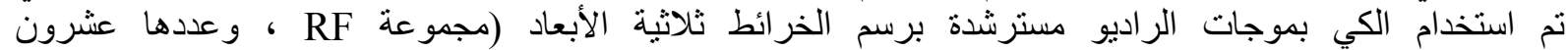
مريضا).و المجموعة الثانية تم استخدام طريقة الكي بالتبريد بها مستخدمين الجيل الثاني من بالونات التئية التبريد وبلغ عددها

أيضا عشرون مريضا(مجموعة CB).

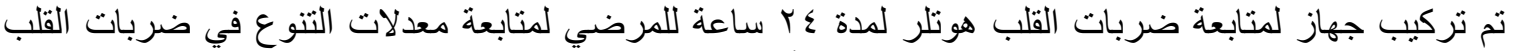

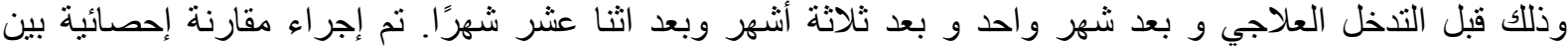
المجمو عتين فيما يتعلق بمعدلات التقلب في ضربات القلات القلب و النتائج الاكلينيكية ولئي المرضي.

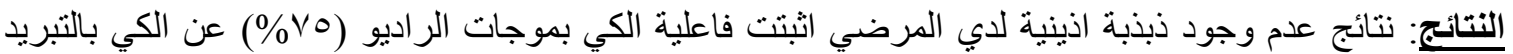

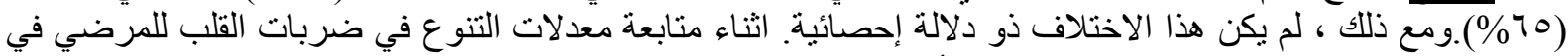

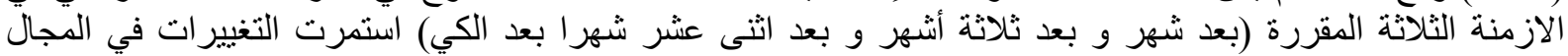

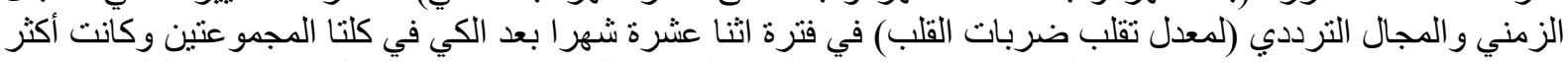

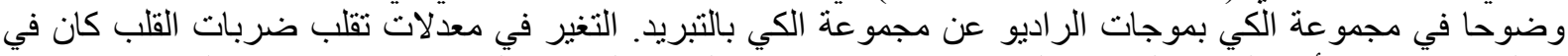

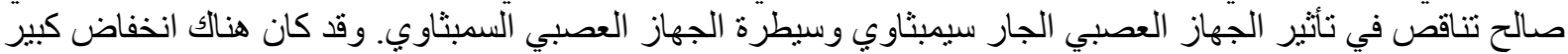
في rMSSD و و المؤشر الثنلاثي (TI) و من ناحية أخرى كانت هناك زيادة كبيرة في (LF/HF).

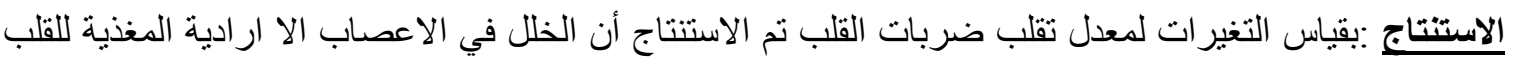

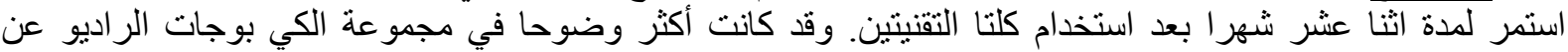
مجمو عة الكي بالتبريد.

الكلمات الدلالية: * كي * عدم انتظام ضربات القلب * الأذين * الذبذبة الأذينية * ضعف الجهاز العصبي اللاإرادي المغذي للقلب * معدل تقلب ضربات القي القب * الكي بالتبريد. 